\title{
Underutilisation of public access defibrillation is related to retrieval distance and time-dependent availability
}

\author{
Charles D Deakin, ${ }^{1,2}$ Steve Anfield, ${ }^{1}$ Gillian A Hodgetts ${ }^{1}$
}

\begin{abstract}
- Additional material is published online only. To view please visit the journal online (http://dx.doi.org/10.1136/ heartjnl-2018-312998).
\end{abstract}

${ }^{1}$ South Central Ambulance Service NHS Foundation Trust, Southampton, UK

${ }^{2} \mathrm{NIHR}$ Respiratory Biomedical Research Unit, Southampton University Hospital, Southampton, UK

\section{Correspondence to}

Professor Charles D Deakin, NIHR Respiratory Biomedical Research Unit, Southampton University Hospital,

Southampton S016 6YD, UK; charlesdeakin@doctors.org.uk

Received 17 January 2018 Revised 17 March 2018

Accepted 25 March 2018 Published Online First 14 May 2018

\section{SLinked}

- http://dx.doi.org/10.1136/ heartjnl-2018-313298

Check for updates

To cite: Deakin $C D$, Anfield S, Hodgetts GA. Heart 2018:104:1339-1343.

\section{ABSTRACT \\ Introduction Public access defibrillation doubles the} chances of neurologically intact survival following outof-hospital cardiac arrest (OHCA). Although there are increasing numbers of defibrillators (automated external defibrillator (AEDs)) available in the community, they are used infrequently, despite often being available. We aimed to match OHCAs with known AED locations in order to understand AED availability, the effects of reduced AED availability at night and the operational radius at which they can be effectively retrieved. Methods All emergency calls to South Central Ambulance Service from April 2014 to April 2016 were screened to identify cardiac arrests. Each was mapped to the nearest AED, according to the time of day. Mapping software was used to calculate the actual walking distance for a bystander between each OHCA and respective $A E D$, when travelling at a brisk walking speed (4 mph).

Results 4012 cardiac arrests were identified and mapped to one of 2076 AEDs. All AEDs were available during daytime hours, but only 713 at night (34.3\%).

$5.91 \%$ of cardiac arrests were within a retrieval (walking) radius of $100 \mathrm{~m}$ during the day, falling to $1.59 \%$ out-of-hours. Distances to rural AEDs were greater than in urban areas $(P<0.0001)$. An AED could potentially have been retrieved prior to actual ambulance arrival in $25.3 \%$ cases.

Conclusion Existing AEDs are underused; $36.4 \%$ of OHCAs are located within $500 \mathrm{~m}$ of an AED. Although more AEDs will improve availability, greater use can be made of existing AEDs, particularly by ensuring they are all available on a $24 / 7$ basis.

\section{INTRODUCTION}

The most effective method of improving the 10\% survival rate from the 275000 annual European out-of-hospital cardiac arrests (OHCAs) ${ }^{1}$ is to strengthen the early links in the chain of survival, particularly bystander cardiopulmonary resuscitation (CPR) and public access defibrillation (PAD). Bystander automated external defibrillator (AED) use doubles overall survival ${ }^{2}$ and may also be of benefit through the delivery of voice prompts and the encouragement to perform CPR, irrespective of shock delivery. ${ }^{3}$

Utilisation of public access AEDs prior to ambulance arrival is only $2 \%-5 \% .^{34}$ The reasons for this remain unclear but are related to three factors: physical availability of a defibrillator, knowledge of its location and public confidence to use the AED. ${ }^{5}$

The aim of this study was to therefore undertake a detailed analysis of OHCA, to identify the overall distribution of AEDs in relation to OHCAs, both by actual walking distance and by retrieval times. We also aimed to establish the effects of reduced AED availability at night and compare potential retrieval times with actual ambulance response times.

\section{METHODOLOGY}

\section{Study design}

This retrospective observational cohort study examined all emergency calls made to South Central Ambulance Service (SCAS) to identify OHCA calls, as categorised by telephone triage (using National Health Service (NHS) Pathways release versions 7, 8, 9 and 10) from 1 April 2014 to 29 April 2016. Each call was then mapped to the nearest available AED.

\section{Study setting}

SCAS covers 3554 sq. miles $\left(9205 \mathrm{~km}^{2}\right)$ and encompasses a residential population of 4 million inhabitants in mixed urban and rural areas. The service receives approximately 500000 emergency and urgent calls annually.

\section{PAD locations}

SCAS maintains a database of 2076 community AEDs, which is available to ambulance call takers and the public through the 'Save a life' app (http:// www.scas.nhs.uk/news/campaigns/savealife). Not all AEDs are available 24/7, but the database does not detail access times. We therefore classified AEDs to either 24/7 availability (eg, cabinet on an external building of a wall or known 24/7 facility), or daytime-only availability (assumed to be approximately $08: 00-18: 00$ ) if they were located in what we termed a static site (eg, doctor's surgery, shopping centre and commercial building).

For cardiac arrests occurring between 08:00 and 18:00, the nearest AED was located. Between 18:00 and 08:00, static AED sites (generally not available at night) were excluded from the calculations.

\section{Mapping software}

PostgreSQL (V.9.5; https://www.postgresql.org) was used to provide a platform onto which all 
OHCA location data, and AED location data were loaded. Urban and rural areas were identified from the 2011 UK Office of National Statistics national census: digital vector boundaries for built-up areas in England and Wales as at 27 March 2011 (Census day) as identified in the Built-up Areas (December 2011) Boundaries V.2 database were added to the PostgreSQL database. (A built-up area refers to a developed area). The OS MasterMap Integrated Transport Network Layer and OS MasterMap Urban Paths Layer were added and linked in order to allow seamless routing between all types of road and footpaths. The shortest walking route from each OHCA to the nearest AED using pgRouting software (V.2.0; http://pgrouting.org) was calculated. Data were then checked for each individual cardiac arrest/AED pairing, using QGIS software (V.1.4; https://www.qgis.org/en/site).

\section{Main outcome measures}

We conducted a series of analyses to evaluate the potential role of public access AEDs in our system. Specifically, we assessed:

1. The proportion of arrests that were within a given distance of the OHCA location according to the time of day.

2. The proportion of arrests that were within a given distance of the OHCA location in both urban and rural locations.

3. The potential time taken to retrieve the nearest AED compared with the actual ambulance response time.

4. The difference in average distance using the pedestrian walking distance versus the straight line method.

The walking distance and time between each OHCA and the nearest AED was calculated for all cardiac arrests. Estimated walking times were calculated using a brisk walking speed of $4 \mathrm{mph}$ (approximately $100 \mathrm{~m} / \mathrm{min}$ ). There is no published data giving the time taken on an emergency call to pass AED location details to a bystander, which we estimated as $2 \mathrm{~min}$. We therefore estimated total retrieval times by allowing for $2 \mathrm{~min}$ to pass the AED location details to a bystander, an additional minute to physically remove the defibrillator from the cabinet (or similar), and a walking speed of $4 \mathrm{mph}$, that is, walking time $+3 \mathrm{~min}$.

\section{Ambulance service AED operational radius}

Between September and November 2017, we surveyed all UK ambulance Trusts to ascertain their operational AED retrieval radius. The method by which these data were applied to each $\mathrm{OHCA} /$ nearest AED pairing was established.

\section{Statistical analysis}

All primary analyses were made using a true walking distance between OHCA and AED rather than a linear 'straight line' route. Demographics details were described using simple non-parametric descriptive statistics. Time-to-access AEDs was compared using a Mann-Whitney U test. A Wilcoxon signed-rank test was used to analyse data comparing walking versus linear routes between cardiac arrest and nearest PAD, with a level of significance set at $5 \%$.

Statistical analyses and generation of graphs were performed using Prism 7 for Mac OSX V.7.0d (GraphPad Software).

\section{RESULTS}

A total of 4169 emergency calls classified as OHCA in the South Central area were identified. Data were insufficient for analysis in 39 of these calls, giving a total of 4130 calls used for data analysis.

Demographic details for this population are as follows:

- 1.1\% of OHCA patients were aged $<15$ years, $31.1 \%$ were aged $15-64$ years and $66.7 \%$ were aged 65 years and over.

- $62 \%$ of the OHCAs treated were male (ratio 1.67:1).

- The proportion of cases that received bystander CPR was $28.1 \%$.

- Emergency Medical Services (EMS) arrived within $8 \mathrm{~min}$ of the emergency call for $73.1 \%$ of patients; the median response time was $6.6 \mathrm{~min}$.

- Initial rhythm was asystole 74.2\%, ventricular fibrillation/ ventricular tachycardia (VF/VT) 14.2\%, pulseless electrical activity (PEA) 9.4\%, bradycardia $0.1 \%$ and unknown $2.2 \%$.

- Approximately $77 \%$ of OHCAs occurred inside the home and 23\% elsewhere.

Of these 4130 calls, a further 118 (2.83\%) were either OHCAs located in a neighbouring ambulance area where we did not have AED data, or incidents where the software could not identify the nearest public route.

Of the 2076 AEDs listed in the database, all were available during daytime hours, but only 713 at night (34.3\%).

\section{Distance to nearest AED: time of day}

The distance to the nearest available AED was plotted for each individual cardiac arrest (table 1 and figure 1). 103/1743 (5.91\%) cardiac arrests were within a retrieval radius (walking route) of $100 \mathrm{~m}$ during the day, falling to $36 / 2269$ (1.59\%) out-of-hours.

Overall, there was a $60.3 \%$ reduction in defibrillator availability out-of-hours $(\mathrm{P}<0.0001)$. In $1491 / 2269$ (65.7\%) cases occurring out-of-hours, the nearest AED was a static (unavailable) defibrillator.

Table 1 Percentage of AEDs at any given radius from an OHCA during the day (08:00-18:00) and night (out-of-hours; 18:00-08:00)

\begin{tabular}{lcc}
\hline Distance to nearest AED & Day $(\mathbf{n = 1 7 4 3 )}(\%)$ & Night $(\mathbf{n}=\mathbf{2 2 6 9 )}(\%)$ \\
\hline$<100 \mathrm{~m}$ & 5.9 & 1.6 \\
$<200 \mathrm{~m}$ & 11.6 & 3.8 \\
$<300 \mathrm{~m}$ & 18.9 & 6.4 \\
$<500 \mathrm{~m}$ & 36.4 & 14.5 \\
$<1000 \mathrm{~m}$ & 68.6 & 37.5 \\
$<2000 \mathrm{~m}$ & 92.4 & 70.8 \\
$<3000 \mathrm{~m}$ & 97.5 & 90.5 \\
$>3000 \mathrm{~m}$ & 100.0 & 100.0 \\
\hline
\end{tabular}

AED, automated external defibrillator; OHCA, out-of-hospital cardiac arrest.

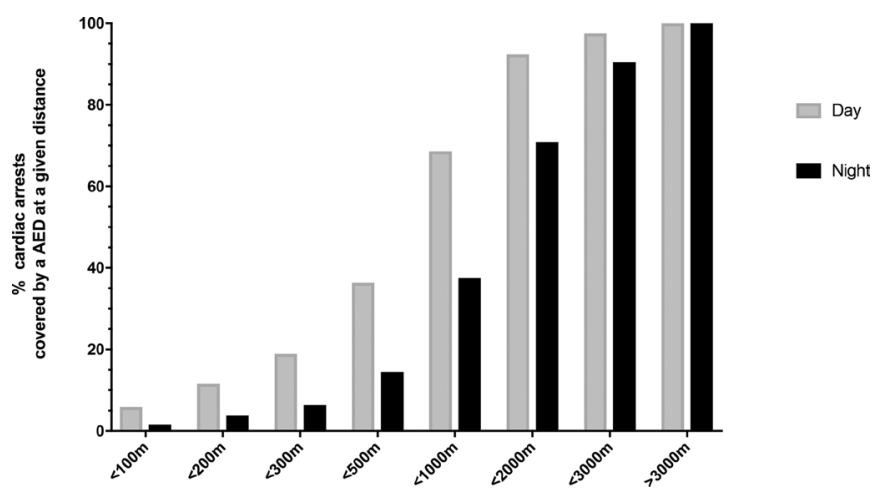

Figure 1 The percentage of cardiac arrests covered by an AED at any given distance, for both daytime (08:00-18:00) and out-of-hours (18:00-08:00) incidents. AEDs, automated external defibrillators. 


\section{Distance to nearest AED: urban versus rural locations}

$145 / 2830(5.1 \%)$ of cardiac arrests were within a walking retrieval distance of $100 \mathrm{~m}$ in urban areas and 68/1182 (5.8\%) in rural areas. Results are shown graphically in figure 2. 1945/2839 $(68.5 \%)$ defibrillators in urban areas were located at static sites (generally not available out-of-hours) compared with 598/1182 $(50.6 \%)$ in rural areas.

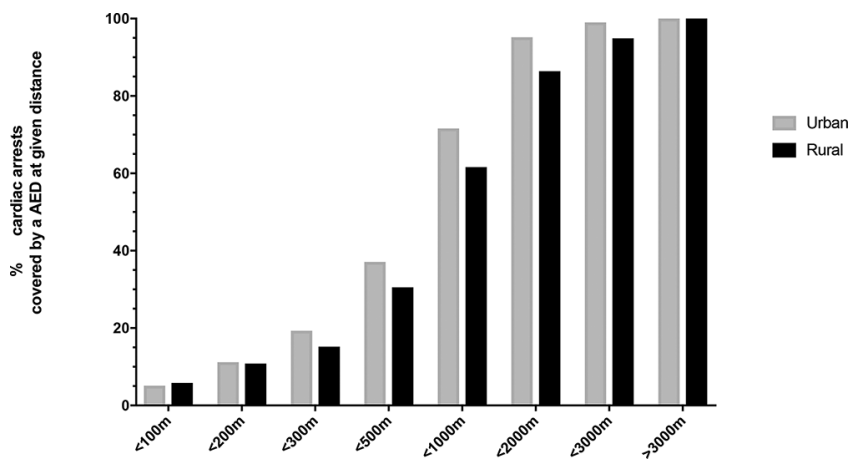

Figure 2 The percentage of cardiac arrests covered by an AED at any given distance, for both urban and rural incidents. AED, automated external defibrillator.

\section{Time to retrieve nearest AED}

Each individual cardiac arrest was mapped to the nearest known AED, available at the time of the incident. An AED could be brought to scene ahead of the ambulance arrival in a total of $1046 / 4130$ calls $(25.3 \%)$. The overall median time in which an ambulance arrived more quickly than theoretical AED retrieval was $5.0 \mathrm{~min}$. The results are shown in figure 3.

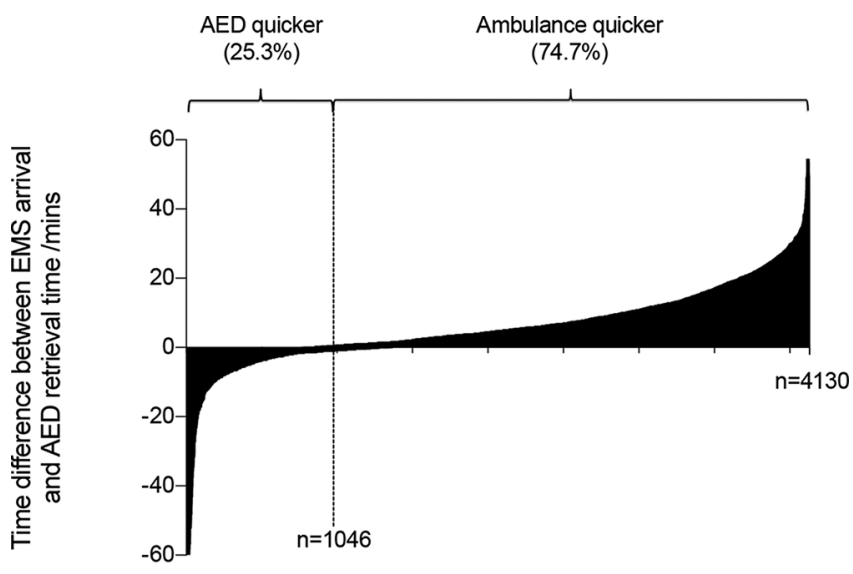

Cardiac arrests ranked by time difference

Figure 3 Time difference between ambulance arrival and potential AED retrieval. (Calls are ranked according to time difference.) AED, automated external defibrillator.

\section{Radial linear versus actual walking routes}

The median walking route distance was $1361 \mathrm{~m} \mathrm{(95 \%} \mathrm{CI} 1506$ to 1570 ) compared with a median linear route distance of $1022 \mathrm{~m}$ that we also calculated (95\% CI 1224 to 1284$)$; $\mathrm{P}<0.0001$. Conventional methodology using linear estimates of distance to the nearest AED therefore underestimates the actual retrieval distance by approximately one-third (33.2\%), effectively underestimating by a similar factor, the time needed to retrieve the nearest AED.

\section{Ambulance service AED retrieval radius}

The results are shown in table 2. All English ambulance Trusts used a linear radial distance overlaid on mapping software to identify the nearest AEDs to an OHCA.

Table 2 AED retrieval radius used by all English ambulance Trusts

\begin{tabular}{ll}
\hline Ambulance Trust & Radius of AED use \\
\hline Isle of Wight & $200 \mathrm{~m}$ but may consider extension \\
\hline East of England & $200 \mathrm{~m}$, recently extended to $400 \mathrm{~m}$ \\
\hline North West Ambulance & $200 \mathrm{~m}$ \\
\hline West Midlands & $200 \mathrm{~m}$ \\
\hline North East & $500 \mathrm{~m}$ \\
\hline London & $100 \mathrm{~m}$ \\
\hline Yorkshire Ambulance & $200 \mathrm{~m}$ static; $600 \mathrm{~m}$ PAD \\
\hline Wales & $500 \mathrm{~m}$ \\
\hline East Midlands & $500 \mathrm{~m}$, with some PADs being $600 \mathrm{~m}$ \\
\hline South West Ambulance Service & $200 \mathrm{~m}$ for all \\
\hline South Central Ambulance Service & $400 \mathrm{~m}$ \\
\hline AED, automated external defibrillator. &
\end{tabular}

\section{DISCUSSION}

This study demonstrates that a lack of defibrillators is often not a rate-limiting step in delivering an AED to an OHCA. During daylight hours when most OHCA occur, we found that approximately one-third of incidents were located within $500 \mathrm{~m}$ of an available AED, and at more than one quarter of all cardiac arrests, an AED could potentially be retrieved prior to ambulance arrival. Our data have not shown as good coverage as that in previous studies. Others have reported $16 \%{ }^{6}-20 \%{ }^{7}$ coverage within $100 \mathrm{~m}$. However, it is likely that this is because of the greater number of private location and rural areas we incorporated, compared with urban-only studies. ${ }^{8}$ Rural areas also have fewer AEDs, which will further reduce overall coverage at any given distance.

Although AEDs were more sparsely distributed in rural areas, there was not a great difference, perhaps because rural communities are more cognisant of the need to provide AED equipment. This is particularly important, because rural ambulance response times are longer than those in urban areas, and AEDs therefore have an even greater potential to improve survival.

One particular area of concern is the lost potential for PAD during out-of-hours periods when AEDs are locked within closed buildings or facilities, as previously reported. ${ }^{9} 10$ Overall, there was a $60.3 \%$ reduction in AED availability out-of-hours, and in two-thirds of cardiac arrests occurring out-of-hours, a static (unavailable) defibrillator was the nearest AED. More AEDs are available out-of-hours in rural areas $(154 / 312 ; 49.4 \%)$ compared with urban areas $(559 / 1773 ; 31.5 \%)$ through mounting them in 24/7 publicly accessible areas. Ensuring that all AEDs were mounted on external walls would have a very significant effect in improving out-of-hours availability.

An important determinant of what is considered an effective operational radius is the distance considered to be reasonable at which to retrieve an AED. Some organisations recommend a maximum distance, while others recommend a maximum time to retrieve the AED. The American Heart Association has previously recommended that '... AEDs should be placed where they can be reached within a short (1 to $1 \frac{1 / 2}{\mathrm{~min}}$ ) brisk walk', ${ }^{11}$ while the Resuscitation Council (UK) currently recommend '...no further than a 2 min brisk walk ...' ${ }^{12}$ At $4 \mathrm{mph}$, this equates to a distance of about $100 \mathrm{~m}$. In the UK, most ambulance services consider that a greater distance is reasonable, recommending between $100 \mathrm{~m}$ and $500 \mathrm{~m}$, with all but one using at least $200 \mathrm{~m}$. As with 


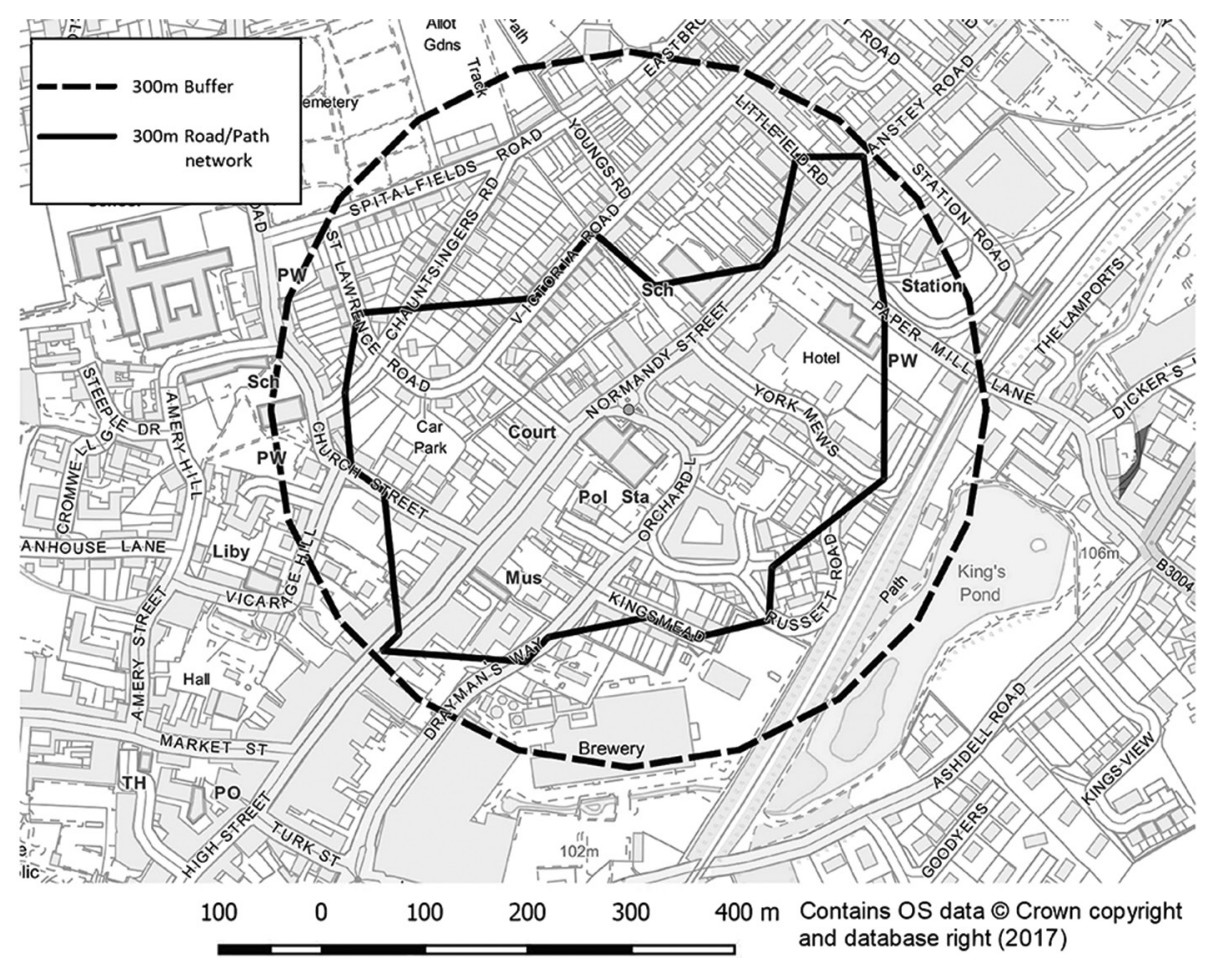

Figure 4 Mapping representation showing the coverage achieved with a $300 \mathrm{~m}$ walking radius compared with the traditional coverage using a linear radius over the same distance.

other studies, we have shown that an AED operational radius of just $100 \mathrm{~m}$ results in relatively few patients having access to an AED, with this number increasing rapidly as the radius expands. Calling ahead to the location of the AED and asking someone to bring it to the scene has the potential to increase the effective operational radius. Further work is required to understand how far and how fast bystanders are capable of travelling to retrieve an AED in order to understand what constitutes an effective operational radius for an AED and produce evidence-based guidelines. The probability of bystander defibrillation decreases rapidly with distance, approximately halving with each additional $100 \mathrm{~m},{ }^{13}$ suggesting that some longer operational radii used by the ambulance services may be optimistic or require more encouragement of bystanders to travel longer distances. Linear routing methodology underestimates the actual retrieval distance to the nearest AED by approximately one-third (33.2\%), and therefore studies using this methodology may produce AED retrieval times that are more rapid than can be realistically achieved. ${ }^{14-16}$ Figure 4 demonstrates the difference in walking radius compared with linear radius over the same distance.

Inevitably, a study of this nature will have some limitations. The study assumes that bystanders are able to locate, and know the quickest walking route to, the nearest AED. We hope that the ambulance service will be able to immediately provide these details, but local knowledge, visible AED signposts and the use of mobile phone apps may all assist in directing bystanders to the nearest AED. We did not have details of the opening hours for buildings that housed AEDs, so broadly divided our AED database into daytime and out-of-hours availability, assuming that outside normal office hours, most AEDs would not be accessible. In practice, opening times are often different during each weekday and certainly at weekends, so without presenting the hourly availability of a 24/7 basis, some degree of averaging is necessary to present the results; doing so does not change the overall conclusions of the study. The ability to retrieve an
AED also assumes that there are sufficient bystanders to enable dispatch of someone to do this. We are not aware of any studies indicating how often a bystander at a cardiac arrest may be available for this task. We have also assumed that in retrieving an AED, a bystander is able to travel at $4 \mathrm{mph}$ but in many cases, this may be unrealistic if the bystander is elderly or unfit. We did not examine the final diagnosis of each cardiac arrest call, but the sensitivity and specificity of NHS Pathways to correctly identify cardiac arrest has previously been documented. ${ }^{17}$

\section{Key messages}

What is already known on this subject?

- Use of an automated external defibrillator (AED) prior to ambulance arrival doubles the chances of neurologically intact survival from cardiac arrest but only occurs in about $2 \%$ of cases. The growing numbers of AEDs has resulted in very little improvement in AED use, and the reasons for this are unclear.

\section{What might this study add?}

- Relatively few AEDs are available within a $100 \mathrm{~m}$ radius of a cardiac arrest, but coverage increases rapidly, with $36 \%$ of cardiac arrests being within a $5 \mathrm{~min}$ retrieval radius. Nighttime availability falls rapidly, as two-thirds of AEDs are not in externally mounted cabinets where 24/7 access is possible.

\section{How might this impact on clinical practice?}

- There is currently considerable potential to improve bystander defibrillation rates by better utilisation of existing devices. Installation of further AEDs will only improve patient outcome if the issues that currently prevent their effective use are addressed. 
In summary, we have demonstrated that although further AEDs are required to improve overall coverage, there is currently considerable potential to improve bystander defibrillation rates using existing AEDs. Installation of further AEDs will only improve patient outcome if the issues that currently prevent the effective use of these existing AEDs are addressed.

Contributors CDD conceived the work. Data were collected by SA and GAH. Data analysis and interpretation was performed by CDD and SA. All authors drafted the article, critically revised it and gave final approval of the version to be published.

Funding This study was funded by a research grant from the Resuscitation Council (UK).

Competing interests CDD has received grants from the Resuscitation Council (UK) of which he is a member and is the ILCOR domain lead for 'Defibrillation'. All authors are employees of South Central Ambulance Service.

Patient consent Not required.

Ethics approval The National Health Service classified this study as a service evaluation and as such does not require ethics approval.

Provenance and peer review Not commissioned; externally peer reviewed.

Data sharing statement The authors will share non-patient identifiable data for the purposes of verification of the results of this study.

(c) Article author(s) (or their employer(s) unless otherwise stated in the text of the article) 2018. All rights reserved. No commercial use is permitted unless otherwise expressly granted.

\section{REFERENCES}

1 Berdowski J, Berg RA, Tijssen JG, et al. Global incidences of out-of-hospital cardiac arrest and survival rates: Systematic review of 67 prospective studies. Resuscitation 2010;81:1479-87.

2 Holmberg MJ, Vognsen M, Andersen MS, et al. Bystander automated external defibrillator use and clinical outcomes after out-of-hospital cardiac arrest: A systematic review and meta-analysis. Resuscitation 2017;120:77-87.

3 Smith CM, Lim Choi Keung SN, Khan MO, et al. Barriers and facilitators to public access defibrillation in out-of-hospital cardiac arrest: a systematic review. Eur Heart $J$ Qual Care Clin Outcomes 2017;3:264-73.
4 Deakin CD, Shewry E, Gray HH. Public access defibrillation remains out of reach for most victims of out-of-hospital sudden cardiac arrest. Heart 2014;100:619-23.

5 Brooks $B$, Chan S, Lander $\mathrm{P}$, et al. Public knowledge and confidence in the use of public access defibrillation. Heart 2015:101:967-71.

6 Sun CL, Demirtas D, Brooks SC, et al. Overcoming Spatial and Temporal Barriers to Public Access Defibrillators Via Optimization. J Am Coll Cardiol 2016;68:836-45.

7 Chan TC, Li H, Lebovic G, et al. Identifying locations for public access defibrillators using mathematical optimization. Circulation 2013;127:1801-9.

8 Siddiq AA, Brooks SC, Chan TC. Modeling the impact of public access defibrillator range on public location cardiac arrest coverage. Resuscitation 2013:84:904-9.

9 Hansen CM, Wissenberg M, Weeke P, et al. Automated external defibrillators inaccessible to more than half of nearby cardiac arrests in public locations during evening, nighttime, and weekends. Circulation 2013;128:2224-31.

10 Sun CL, Brooks SC, Morrison LJ, et al. Ranking Businesses and Municipal Locations by Spatiotemporal Cardiac Arrest Risk to Guide Public Defibrillator Placement. Circulation 2017; 135:1104-19.

11 Aufderheide T, Hazinski MF, Nichol G, et al. Community lay rescuer automated external defibrillation programs: key state legislative components and implementation strategies: a summary of a decade of experience for healthcare providers, policymakers, legislators, employers, and community leaders from the American Heart Association Emergency Cardiovascular Care Committee, Council on Clinical Cardiology, and Office of State Advocacy. Circulation 2006;113:1260-70.

12 Resuscitation Council (UK) and British Heart Foundation. A guide to Automated External Defibrillators (AEDs). 2017.

13 Sondergaard KB, Hansen SM, Pallisgaard JL, et al. Out-of-hospital cardiac arrest: Probability of bystander defibrillation relative to distance to nearest automated external defibrillator. Resuscitation 2018;124:138-44.

14 Hazinski MF, Markenson D, Neish S, et al. Response to cardiac arrest and selected life-threatening medical emergencies: the medical emergency response plan for schools: A statement for healthcare providers, policymakers, school administrators, and community leaders. Circulation 2004;109:278-91.

15 Hansen CM, Lippert FK, Wissenberg M, et al. Temporal trends in coverage of historical cardiac arrests using a volunteer-based network of automated external defibrillators accessible to laypersons and emergency dispatch centers. Circulation 2014:130:1859-67.

16 Fredman D, Svensson L, Ban Y, et al. Expanding the first link in the chain of survival - experiences from dispatcher referral of callers to AED locations. Resuscitation 2016;107:129-34.

17 Deakin CD, England S, Diffey D. Ambulance telephone triage using 'NHS Pathways' to identify adult cardiac arrest. Heart 2017;103:738.2-44. 